\title{
High-Speed Photography of Human Trabecular Bone during Compression
}

\author{
Philipp J. Thurner ${ }^{1}$, Blake Erikson ${ }^{1}$, Zachary Schriock ${ }^{1}$, John Langan ${ }^{2}$, Jeff Scott $^{2}$, Maria Zhao $^{2}$, \\ Georg E. Fantner ${ }^{1}$, Patricia Turner ${ }^{1}$, Johannes H. Kindt ${ }^{1}$, Georg Schitter ${ }^{1}$, Paul K. Hansma ${ }^{1}$ \\ ${ }^{1}$ University of California Santa Barbara, Santa Barbara, CA, USA \\ ${ }^{2}$ Computational Sensors Corp., Santa Barbara, CA, USA
}

\begin{abstract}
The mechanical properties of healthy and diseased bone tissue are extensively studied in mechanical tests. Most of this research is motivated by the immense costs of health care and social impacts due to osteoporosis in post-menopausal women and the aged. Osteoporosis results in bone loss and change of trabecular architecture, causing a decrease in bone strength. To address the problem of assessing local failure behavior of bone, we combined mechanical compression testing of trabecular bone samples with high-speed photography. In this exploratory study, we investigated healthy, osteoarthritic, and osteoporotic human vertebral trabecular bone compressed at high strain rates simulating conditions experienced in individuals during falls. Apparent strains were found to translate to a broad range of local strains. Moreover, strained trabeculae were seen to whiten with increasing strain. We hypothesize that the effect seen is due to microcrack formation in these areas, similar to stress whitening seen in synthetic polymers. From the results of a motion energy filter applied to the recorded movies, we saw that the whitened areas are, presumably, also of high deformation. We believe that this method will allow further insights into bone failure mechanisms, and help toward a better understanding of the processes involved in bone failure.
\end{abstract}

\section{INTRODUCTION}

Mechanical testing of trabecular bone is a vast experimental field ${ }^{1}$, mainly motivated by the cost and social impact of osteoporosis, a systemic, skeletal disease ${ }^{2}$, which comes with a reduction of bone strength and a concomitant increase of fracture risk. Trabecular bone is situated at the end of the long bones and in the spinal column, where it fills all of the inner vertebral space. In the long bones it transfers loads from joint faces onto the midshaft of the bone; in lumbar vertebrae, trabecular bone carries and transfers about $90 \%$ of the applied load. Thus a change in trabecular bone quality can have a huge impact on strength and on fracture risk in vertebrae. Standard mechanical tests deliver, however, only integral information on a bone sample and no information of local processes experienced in the elastic, yield, and post-yield region. In order to close this gap, experiments have been devised to combine mechanical testing and imaging of trabecular and also cortical bone $\mathrm{e}^{3,4,5,6,7}$. Most of these approaches deliver 3D information. However, they all are somewhat time consuming and thus limited to quasi-static testing and/or recording of only a few different states of a sample subjected to mechanical testing. In contrast high-speed photography is designed to record very fast processes, which allows uncovering in a 2D fashion the failure dynamics of bone samples subjected to mechanical testing in real time. In this communication we present a novel system consisting of a custom made mechanical testing device and a high-speed camera used to record movies of small bone samples loaded in compression at strain rates around $0.5 \mathrm{~s}^{-1}$. In an exploratory study we used this system to investigate failure of healthy osteoarthritic and osteoporotic trabecular bone. During compression 
all samples exhibited whitening of strongly deformed trabeculae, which is hypothesized to be due to microcracking ${ }^{8}$. Applying a motion energy filter we present an example showing correlation between local deformation as evidenced by detected motion and whitening. Overall, high-speed photography was found to be beneficial delivering complementary results to conventional mechanical tests.

\section{EXPERIMENTAL DETAIL}

Sample Preparation: In the presented study we investigated three bone samples from a healthy male donor 21 years of age, an osteoarthritic female donor 65 years of age, and an osteoporotic female donor 85 years of age. Cuboid bone samples with a height of $4 \mathrm{~mm}$ and cross-sectional dimensions of $4.9 \mathrm{~mm}$ by $4.9 \mathrm{~mm}$ were cut from vertebrae using a bandsaw (Marmed Inc. Cleveland, OH, USA) under constant irrigation with water. The smaller sample dimension is oriented in the original principal load bearing direction. Bone marrow was extracted from the specimens using a jet of pressurized water.

Mechanical Testing: A photograph of the experimental setup including the high-speed camera system is given in Figure 1A. Compression testing of all samples was done using a custom-made mechanical testing device, consisting of a load frame and a piston driven by a stepper motor. The piston impinges onto the transparent sample chamber made from PMMA consisting of a frame and a plunger, which applies the displacement onto the sample. The sample chamber has a fluid inlet and outlet, allowing for full immersion of the specimen in fluid. In this experiment, the displacement of the plunger, and from this the apparent strain, was directly calculated from the recorded high-speed photography frames (cp. Subsection on Image Processing). The load on the sample was measured with a $250 \mathrm{lb}$ capacity load cell (Model LBC-250, Transducer Techniques Inc., Temecula, Ca, USA), which was mounted onto the lower end of the piston. The experiment was controlled using a PC and LabView (National Instruments, Austin, TX, USA). A displacement rate of $2 \mathrm{~mm} / \mathrm{s}$ was used for all mechanical tests resulting in strain rates around $0.5 \mathrm{~s}^{1}$. From the stress strain curves elastic modulus, yield stress and strain, as well as failure stress and strain were calculated.

High-Speed Photography: We used an Ultima 512 high-speed camera (Photron Inc., San Diego, $\mathrm{Ca}$, USA) equipped with a KC lens, with a KC-AUX and an IF-3 lens mounted on top of it (Infinity, Boulder, Co, USA). The high-speed camera can record up to $32000 \mathrm{frames} / \mathrm{s}$ but offers limited memory of $500 \mathrm{MB}$. We recorded images at a rate of 1000 frames/s and a shutter opening time of $1 / 8500 \mathrm{~s}$. The scene was illuminated from the front using two fiber lights (MH-100, Dolan Jenner Industries Inc. Lawrence, Ma, USA) at angles of about $\pm 45^{\circ}$.

Image Processing: For the computation of apparent strains we measured the size of the plunger in pixels in the different movies and used this as a calibration for the physical size of an image pixel. A texture correlation ${ }^{9}$ algorithm was then applied to detect the motion of the plunger. A region of interest with high contrast was selected on the plunger and tracked in subsequent recorded frames. The tracking also allowed synchronization between recorded image data and stress curves. A motion energy filter ${ }^{10}$ was applied to a selected region of interest in the highspeed photography movie retrieved from the osteoarthritic bone sample, which detects sample motion. Motion energy processing utilizes spatial and temporal filtering to produce an output image sequence where image intensity represents a measure of local scene motion. Prior to motion detection the dataset was subjected to a spatial frequency filter. 


\section{RESULTS}

The stress-strain curves retrieved from the compression experiments are depicted in Figure 1B. The differences in mechanical properties of the three different bone samples are clearly visible. The mechanical parameters derived from the curves are given in the Table 1. In this preliminary all values decrease from healthy to osteoarthritic and osteoporotic bone. One exception is that the elastic modulus is higher for the osteoporotic sample compared to the osteoarthritic one, which might be an experimental artifact. In terms of fracture resistance, the energy to failure is of special interest, which is drastically reduced in diseased, compared to healthy bone. Selected frames from the movies of the samples under compression recorded with the high-speed photography system are given in Figure 2. The individual frames correspond to the points marked on the stress-strain curves depicted in Figure 1B. The frames show that the apparent strain is distributed quite unevenly across the bone samples, some trabeculae are strongly deformed, whereas others seem not to be deformed at all. Strongly deformed trabeculae can be seen to whiten with increasing strain in all samples; however, here the effect is more pronounced in the healthy and osteoarthritic bone samples. A magnified detail from the latter is also shown in Figure 2, where the whitening effect is clearly visible. The magnified detail of the osteoarthritic sample was processed using a motion energy filter in order to detect absolute and relative motion. The filter was applied in both the $x$ - (left-right) and y-direction (up-down) of the recorded frames. The outcome of the filtering process is a movie containing the detected motion in the different directions over time. Figure 3 shows one frame of the high-speed sequence, the region of interest used for motion filtering as well as the response of the motion energy filter in both directions and a vector plot of the combined motion. For the trabecula that is bent to the left and whitens during compression a clear motion signature into that direction could be detected.

Table 1. Mechanical parameters derived from the recorded stress strain curves.

E...elastic modulus, $\varepsilon_{\mathrm{y}} \ldots$ yield strain, $\sigma_{\mathrm{y}} \ldots$ yield stress, $\varepsilon_{\mathrm{f}} \ldots$ failure strain, $\sigma_{\mathrm{f}} \ldots$ failure stress, $\mathrm{E}_{\mathrm{f}} \ldots$ energy to failure

\begin{tabular}{c|c|c|c|c|c|c} 
Donor & $\mathrm{E}[\mathrm{MPa}]$ & $\varepsilon_{\mathrm{y}}[\% \mid$ & $\sigma_{\mathrm{y}}[\mathrm{MPa}]$ & $\varepsilon_{\mathrm{f}}[\%]$ & $\sigma_{\mathrm{f}}[\mathrm{MPa}]$ & $\mathrm{E}_{\mathrm{f}}[\mathrm{J}]$ \\
\hline \hline Healthy, male, 21 yr & 60.0 & 4.5 & 2.6 & 10.9 & 4.0 & 28.4 \\
Osteoarthritic, female, 65 yr & 37.7 & 2.8 & 1.0 & 6.9 & 1.6 & 6.8 \\
Osteoporotic, female, 85 yr & 46.2 & 1.3 & 0.5 & 4.6 & 0.8 & 2.7
\end{tabular}
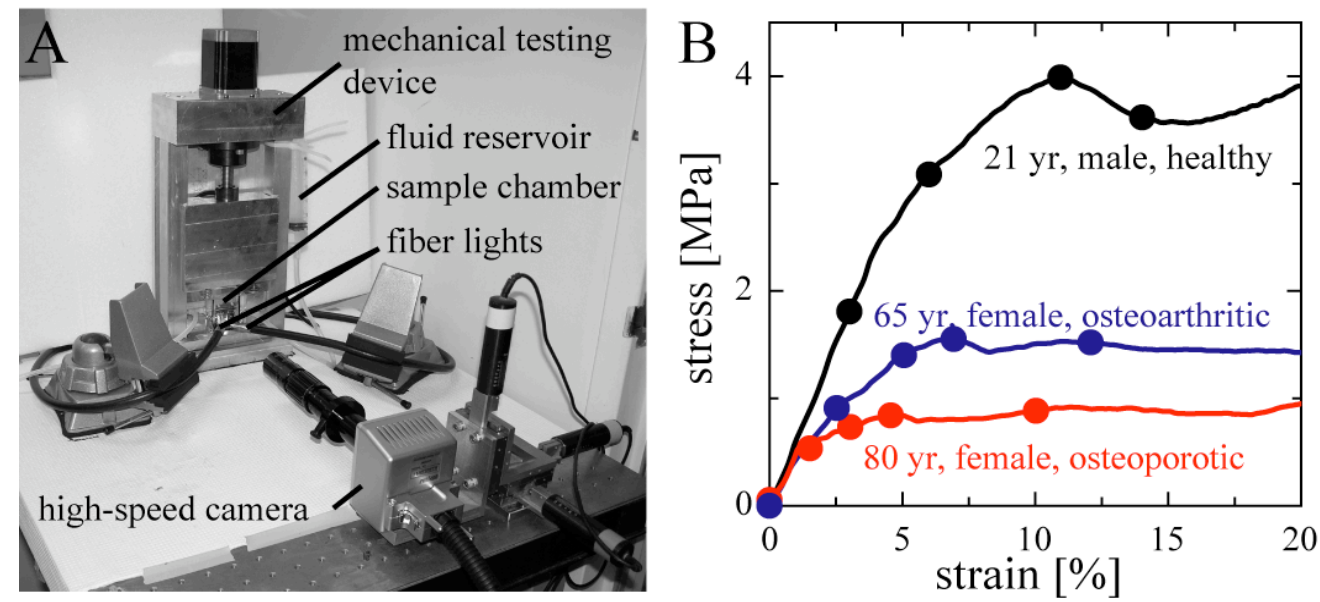

Figure 1. Photograph of the experimental setup (A), and stress vs. strain curves (B) retrieved from the three bone specimens tested in compression. 
male, healthy, $21 \mathrm{yr}$
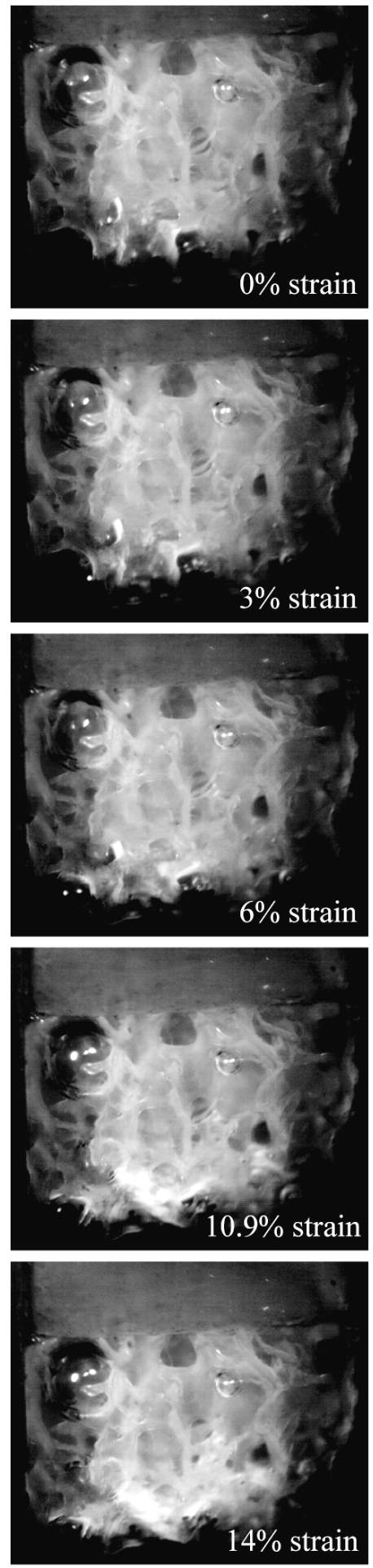

female, osteoarthritic, $65 \mathrm{yr}$
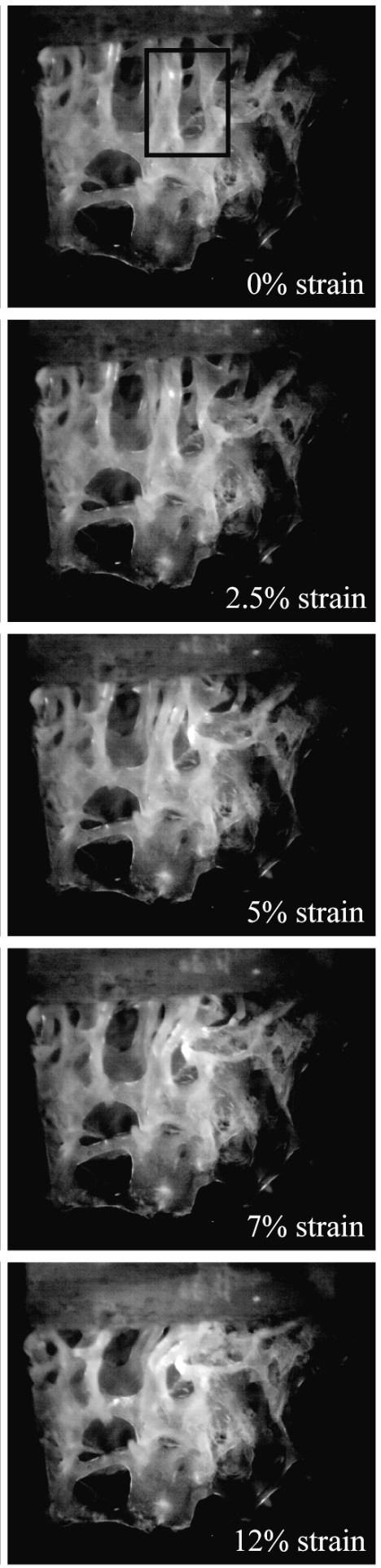

female, osteoporotic, $85 \mathrm{yr}$
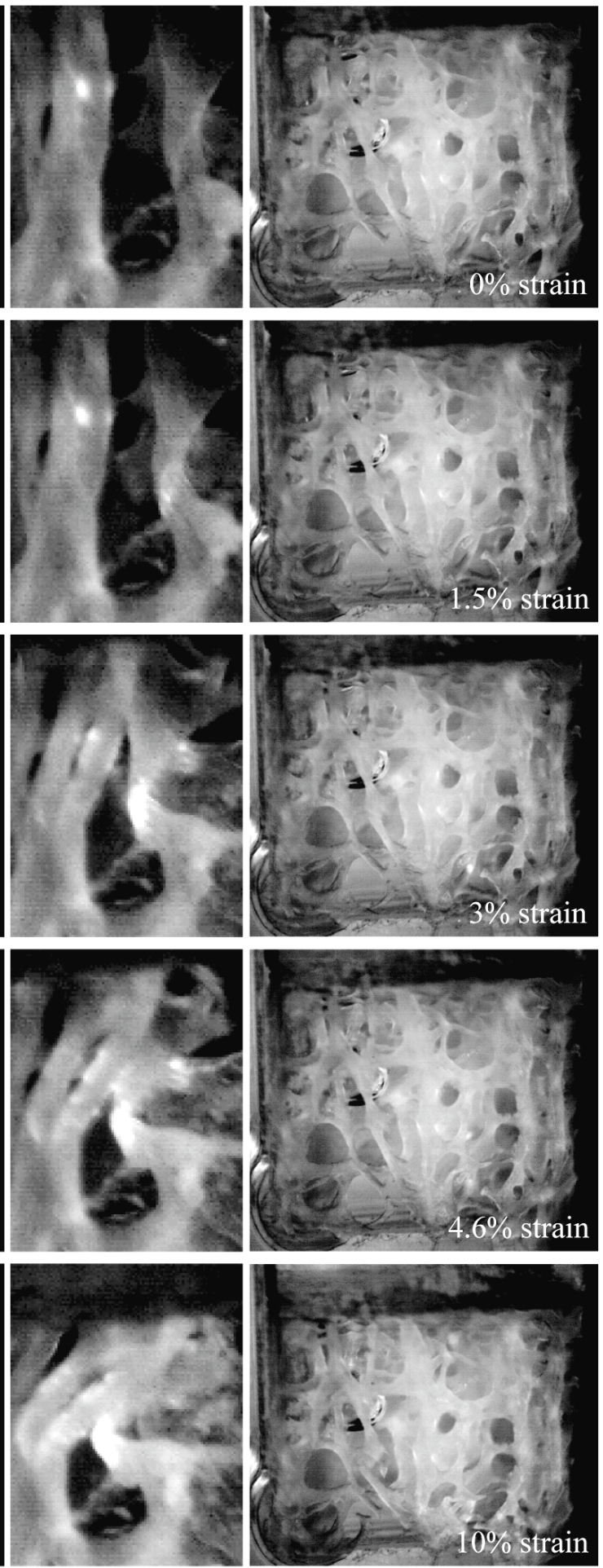

$1 \mathrm{~mm}$

Figure 2. High-speed photography frames of the bone specimens tested in compression. The frames correspond to the points marked on the stress strain curves in Figure 1. Trabeculae are seen to whiten with increasing local strain, especially in the case of the healthy and osteoarthritic bone specimens, as can also be seen in the magnified detail of the osteoarthritic bone sample 


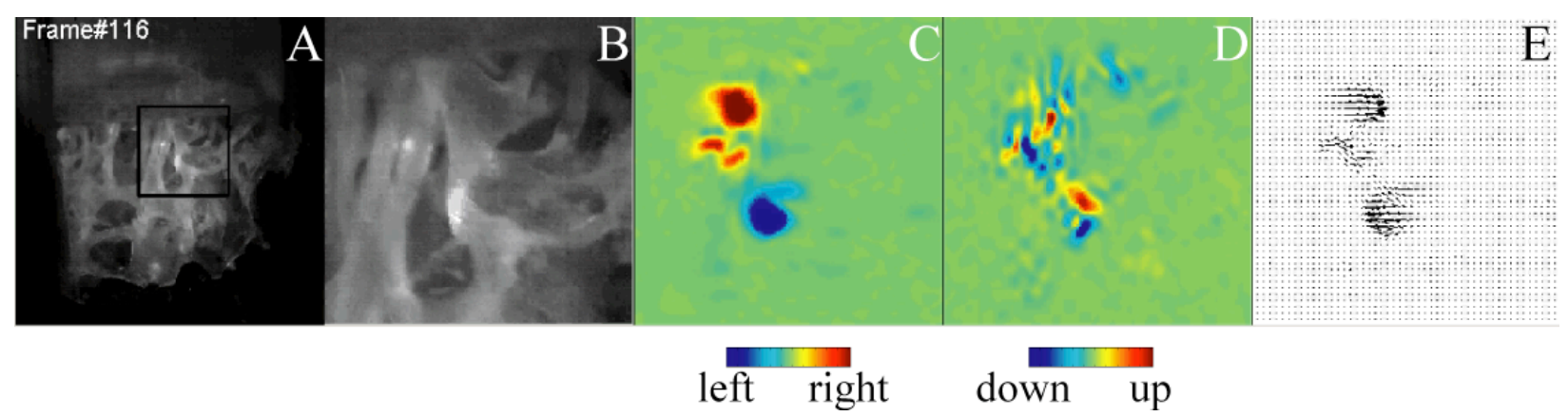

Figure 3. Frame (A) and magnified detail (B) of the osteoarthritic bone sample under compression. The motion energy filter was applied in two directions, horizontal (C), and vertical (D). The combined motion vectors are also given (E).

The fact that the vector plot in this same area Figure 3E does not appear uniform in is an indication for elevated local strains.

\section{DISCUSSION}

The differences in the mechanical properties of the three bone specimens are clearly shown in Figure 1 and Table 1. Differences in mechanical properties of bone are generally associated with sex, age and health of the donor. Bone disease, especially osteoporosis, results in an increase of fracture risk and reduced bone stability. This parameter could be associated with the energy input needed for failure of a bone sample. In our preliminary study this value is drastically reduced going from healthy to osteoarthritic and osteoporotic bone. It has to be noted, that the mechanical tests were subjected to a systematic error, since the lower support for the bone sample in the holder was not planar. Thus the stress values are underestimated. Since the setup was the same for all three samples their comparison is feasible. In addition to the mechanical tests the combination with high-speed photography delivered image data of the failure dynamics in real time revealing local events. In the recorded movies it can be clearly seen that apparent strain is translated to a whole range of deformations. This result is in agreement with the work of Odgaard and others ${ }^{11}$. The retrievable data is two-dimensional, but the acquisition is much faster in contrast to other approaches delivering 3D data, which are usually combined with quasi-static tests $^{3,4,6,7}$. Functional imaging of trabecular bone under load using high-speed photography can be used with a variety of strain rates. Especially high strain rates are accessible with this technique. High-speed photography has previously been applied to monitor spiral fracture of sheep femora ${ }^{12}$ or movements and displacements in functional spine units ${ }^{13}$, but to our best knowledge has not been used to investigate trabecular bone under compressive loads. Recorded high-speed photography frames show that deformed trabeculae whiten with increasing apparent strain. This whitening effect is hypothesized to be due to microdamage ${ }^{8}$, similar to stress whitening detected in polymers ${ }^{14}$. The osteoarthritic bone sample shows this effect most dramatically in this study. A region of interest where we could clearly identify bending and whitening of a trabecula was selected from this data and subjected to a motion energy filter. The filter detected elevated motion of the whitened area with increasing strain, and since the displacement pattern was not uniform this is also an indication for elevated local strains. We hypothesize that the detected whitening and also microdamage appears at points of highest strain, which would be consistent with failure initiation in compact bone, which was found to be at the point of highest local strain ${ }^{15}$. Further research is required to investigate whether whitening is directly related to microdamage and to quantify the output of motion energy filtering. 


\section{CONCLUSION}

In this communication we present an exploratory study combining mechanical testing of trabecular bone with functional imaging using high-speed photography. Healthy male bone was found to have a higher elastic modulus, strength and toughness compared to osteoarthritic and osteoporotic bone, also the yield point was found to be at a higher strain. In compressed bone apparent strain is made up of greatly differing strains at the local level as can be seen in highspeed photography movies. Deformed trabeculae whiten, which increases with increasing apparent and presumably also local strain. This effect is more pronounced in the healthy and osteoarthritic bone samples compared to the osteoporotic bone sample that we tested. Using the osteoarthritic bone sample as an example it was shown that the whitening can be correlated to elevated displacements and qualitatively also to elevated local strains. Further research is required to show whether whitening is conclusively due to microcracking. Overall high-speed photography demonstrates its value as it is non-invasive and can be simply added to a mechanical testing experiment, giving insight into failure dynamics in real time.

\section{ACKNOWLEDGEMENTS}

We are gratefully indebted the SNF Postdoctoral fellowship PBEZ-105116, FWF Project No. J2395-N02, ÖAW DOC-Fellowship, NIH Grant Number GM65354, Veeco/DI Grant Number SB030071, MRL NSF Grant Number DMR00-80034, NASA/URETI Grant Number BiMAt NCC-1-02037, USARL (ICB) Grant Number DAAD19-030D-0004, and USARIEM (ICB) Grant Number W911QY-04-P-0516 for financial support.

\section{REFERENCES}

1

2

T.M. Keaveny and W.C. Hayes, J Biomech Eng 115 (4B), 534 (1993).

L. J. Melton, 3rd, E. A. Chrischilles, C. Cooper et al., Journal of Bone and Mineral Research 7 (9), 1005 (1992); J. A. Kanis, Neth J Med 50 (5), 198 (1997). R. Muller, S. C. Gerber, and W. C. Hayes, Technol Health Care 6 (5-6), 433 (1998). B. K. Bay, T. S. Smith, D. P. Fyhrie et al., Experimental Mechanics 39 (3), 217 (1999). D. P. Nicolella, A. E. Nicholls, J. Lankford et al., Journal of Biomechanics 34 (1), 135 (2001).

A. Nazarian and R. Muller, J Biomech 37 (1), 55 (2004).

P. Thurner, P. Wyss, R. Voide et al., in Developments in X-Ray tomography IV, edited by U. Bonse (Denver, Colorado, 2004), Vol. 5535, pp. 112.

J. D. Currey, Bones: Structure and Mechanics. (Princeton University Press, Princeton, 2002).

B. K. Bay, J Orthop Res 13 (2), 258 (1995).

E. H. Adelson and J. R. Bergen, Journal of the Optical Society of America a-Optics Image Science and Vision 2 (2), 284 (1985).

A. Odgaard, I. Hvid, and F. Linde, J Biomech 22 (8-9), 829 (1989).

W. Bonfield and M. D. Grynpas, Journal of Biomechanics 15 (8), 555 (1982).

A. L. Osvalder, P. Neumann, P. Lovsund et al., Journal of Biomechanics 26 (10), 1227 (1993).

B. W. Cherry and T. S. Hin, Polymer 22 (12), 1610 (1981).

R. K. Nalla, J. H. Kinney, and R. O. Ritchie, Nat Mater 2 (3), 164 (2003). 